\title{
Nobel Lecture: Graphene: Materials in the Flatland*
}

\author{
K. S. Novoselov \\ School of Physics and Astronomy, The University of Manchester, Oxford Road, Manchester \\ M13 9PL, United Kingdom
}

(Received 26 May 2011; published 3 August 2011)

DOI: $10.1103 /$ RevModPhys.83.837

Much like the world described in Abbott's Flatland, graphene is a two-dimensional object. And, as "Flatland" is "a romance of many dimensions," graphene is much more than just a flat crystal. It possesses a number of unusual properties which are often unique or superior to those in other materials. In this brief lecture I would like to explain the reason for my (and many other people's) fascination with this material, and invite the reader to share some of the excitement I've experienced while researching it.

\section{CONTENTS}

Graphene and Its Unusual Properties

A. Two-dimensional crystals

1. Stability of 2D crystals

2. Graphene creation

3. Other $2 \mathrm{D}$ crystals

4. Out to Spaceland: 2D-based heterostructures

B. Chiral quasiparticles and the electronic properties of graphene

1. Linear dispersion relation and chirality

2. The Klein paradox

3. Half-integer quantum Hall effect

4. Effect of mechanical deformation

5. Graphene optics

6. Bilayer graphene

C. Graphene applications

1. Graphene support

2. Transparent conductive coating

3. Graphene transistors

4. Graphene composites

5. Other applications cleavage technique, the Scotch-tape method has a low barrier to entry in that it doesn't require large investments or complicated equipment, which has helped considerably to broaden the geography of graphene science.

Another source of graphene's widespread popularity is that it appeals to researchers from a myriad of different backgrounds. It is the first example of 2D atomic crystals, whose properties from the thermodynamics point of view are significantly different from those of 3D objects. It is also a novel electronic system with unprecedented characteristics (Castro Neto et al., 2009). It can be thought of as a giant molecule which is available for chemical modification (Ruoff, 2008; Loh et al., 2010) and is promising for applications (Geim and Novoselov, 2007; Geim, 2009) ranging from electronics (Geim and Novoselov, 2007; Geim, 2009; Schwierz, 2010) to composite materials (Stankovich et al., 2006; Geim, 2009; Gong et al., 2010). These factors allow for true multi- and cross-disciplinary research. Thanks to these attributes, within 7 years of the first isolation of graphene we have accumulated as many results and approached the problem from as many different perspectives as other areas of science would more commonly achieve over several decades.

The major draw to people in the field, though, is graphene's unique properties, each of which seems to be superior to its rivals. This material is the first $2 \mathrm{D}$ atomic crystal ever known to us (Novoselov et al., 2005a); the thinnest object ever

Graphene is a two-dimensional (2D) atomic crystal which consists of carbon atoms arranged in a hexagonal lattice (Fig. 1). Although sporadic attempts to study it can be traced back to 1859 (Brodie, 1859), active and focused investigation of this material started only a few years ago, after a simple and effective way to produce relatively large isolated graphene samples was found (Novoselov et al., 2004, 2005a). The original "Scotch-tape method" (Novoselov et al., 2004, 2005a) appeared to be so simple and effective that this area of science grew extremely quickly, and now hundreds of laboratories around the world deal with different aspects of graphene research. Also known as the micromechanical

\footnotetext{
* The 2010 Nobel Prize for Physics was shared by Andre K. Geim and K. S. Novoselov. These papers are the text of the address given in conjunction with the award.
}

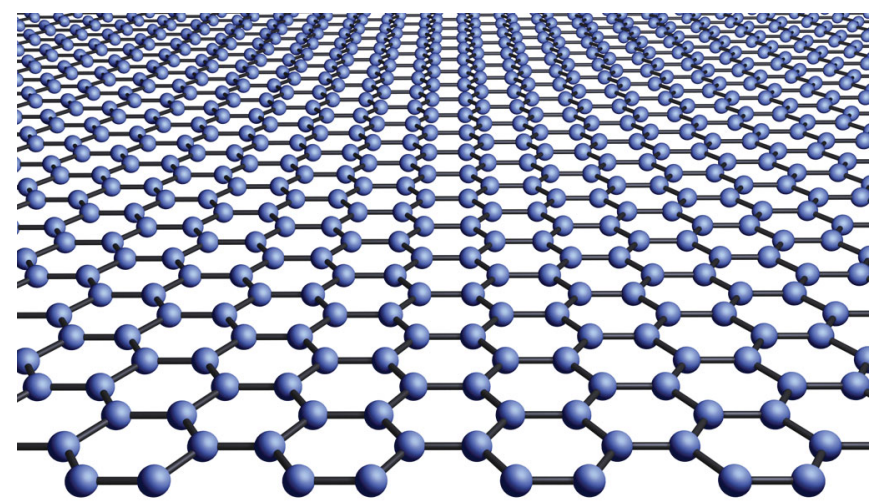

FIG. 1 (color). The crystal structure of graphene-carbon atoms arranged in a honeycomb lattice. 
obtained; the world's strongest material (Lee et al., 2008); its charge carriers are massless Dirac fermions (Novoselov et al., 2005b; Zhang et al., 2005; Geim and Novoselov, 2007); it is extremely electrically (Meric et al., 2008) and thermally (Balandin et al., 2008) conductive; very elastic; and impermeable to any molecules (Bunch et al., 2008) - the list goes on. Even a simple inventory of graphene's superlative qualities would require several pages, and new entries are being added on a monthly basis.

As it is not possible to give a comprehensive overview of all of graphene's properties in one lecture, I will limit myself to just three, which, in my opinion, give the best possible impression of graphene: (i) it is the first example of $2 \mathrm{D}$ atomic crystals, (ii) it demonstrates unique electronic properties, thanks to charge carriers which mimic massless relativistic particles, and (iii) it has promise for a number of applications.

\section{A. Two-dimensional crystals}

\section{Stability of $2 \mathrm{D}$ crystals}

Intuitively one can easily discern the difference between two- and three-dimensional objects: restrict the size or motion of an object to its width and length and forget (or reduce to zero) its height and you will arrive in "flatland." The consequences of subtracting one (or more) dimensions from our 3D world are often severe and dramatic. To give just a few examples: There are no knots in 2D space; the probability of reaching any point in $d$-dimensional space by random walking is exactly unity, for $d=1$ and $d=2$ and smaller than 1 in higher dimensions (Pólya, 1921); the problem of bosons with a repulsive potential in $1 \mathrm{D}$ is exactly equivalent to that of fermions, since particles cannot penetrate each other and cannot be swapped (Girardeau, 1960; Lieb and Liniger, 1963) (the Tonks-Girardeau gas and fermionization of bosons in 1D problem); and it is impossible to have thermodynamic equilibrium between different phases in 1D systems (Landau and Lifshitz, 1980).

Many of the peculiar properties that one can expect in 2D systems are present due to so-called "logarithmic divergences," with the most well-known example being the weak localization quantum corrections to the conductivity in 2D. In particular, a series of works by Peierls (Peierls, 1934, 1935), Landau (Landau, 1937; Landau and Lifshitz, 1980), Mermin (Mermin, 1968) and Wagner (Mermin and Wagner, 1966) demonstrated the theoretical impossibility of longrange ordering (crystallographic or magnetic) in 2D at any finite temperatures. The stability of 2D crystals [here the theory has to be expanded to take flexural phonons or outof-plane displacements into account (Chaikin and Lubensky, 1995; Nelson et al., 2004; Fasolino et al., 2007)] is a simple consequence of divergences at low $k$ vectors, when the integration of the atomic displacements is taken over the whole 2D $k$ space.

It is important to mention that such instabilities are the result of thermal fluctuations and disappear at $T=0$. Also, strictly speaking, at any finite temperature the fluctuations diverge only for infinitely large $2 \mathrm{D}$ crystals $(k \rightarrow 0)$; as the divergences are weak (logarithmic), crystals of limited sizes might exhibit infinitely small fluctuations at least at low temperatures.

These fluctuations place a fundamental restriction on the existence and synthesis of low-dimensional crystals. Growth or synthesis generally requires elevated temperatures-at which only crystallites of very limited size can be stable in the flat form - and, as the bending rigidity of such crystals is usually low, they would generally crumple and fold easily and form 3D structures (which might also help in reducing the energy of unsaturated dangling bonds at the perimeter). The largest flat molecule synthesized to date therefore is $\mathrm{C}_{222}$ (Simpson et al., 2002), and the method used to create it is the low (room) temperature cyclodehydrogenation of a 3D precursor molecule.

A possible way around the problem of 2D crystals' instability is synthesis as part of a 3D structure, with subsequent extraction of the 2D part of the system at low temperatures (in fact, such a strategy is the basis of all methods of graphene synthesizing available to date) (Geim and Novoselov, 2007). The fluctuations, which might diverge at high temperatures, are quenched during the synthesis due to the interaction with the 3D matrix (substrate) whenever the extraction of 2D crystals could be done at low temperatures where the fluctuations are suppressed.

\section{Graphene creation}

The simplest implementation of this method for graphitic materials is to use bulk graphite and exfoliate it into individual planes. Graphite is a layered material and can be considered as a stack of individual graphene layers. High-quality graphite typically requires growth temperatures of above $3000 \mathrm{~K}$, but exfoliation can be done at room temperatures - an order of magnitude lower than the growth temperatures. In fact, many of us have performed this procedure numerous times while using pencils, as drawing with a pencil relies on exfoliation of graphite (though not up to the monolayer limit, which would be practically invisible to the naked eye).

Graphite exfoliation techniques slightly more elaborate than writing with a pencil have been attempted by several groups (Mizushima et al., 1971; Fujibayashi, 1973; Fujibayashi and Mizushima, 1973; Ebbesen and Hiura, 1995; Ohashi et al., 1997; Lu et al., 1999; Gan et al., 2003) and thin graphitic films have been obtained. But even graphitic films only 20 layers thick would generally behave similarly to bulk graphite, so the real breakthrough came when monolayer films of graphene, large enough to be studied by conventional techniques, were obtained (Novoselov et al., 2004, 2005a). The technique used in those cases is known as the micromechanical cleavage or Scotchtape method (Fig. 2). The top layer of the high-quality graphite crystal is removed by a piece of adhesive tape, which — with its graphitic crystallites-is then pressed against the substrate of choice. If the adhesion of the bottom graphene layer to the substrate is stronger than that between the layers of graphene, a layer of graphene can be transferred onto the surface of the substrate, producing extremely highquality graphene crystallites via an amazingly simple procedure. In principle, this technique works with practically any surface which has reasonable adhesion to graphene. 

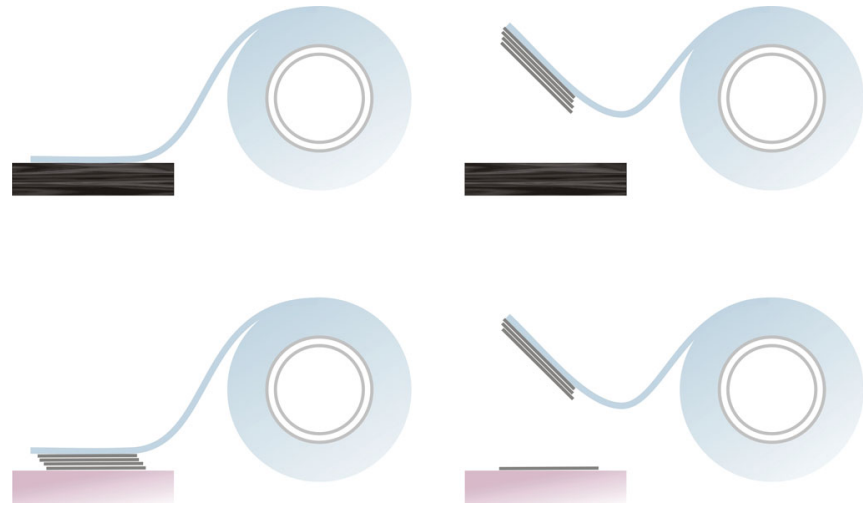

FIG. 2 (color). The micromechanical cleavage technique ("Scotch-tape" method) for producing graphene. Top row: Adhesive tape is used to cleave the top few layers of graphite from a bulk crystal of the material. Bottom left: The tape with graphitic flakes is then pressed against the substrate of choice. Bottom right: Some flakes stay on the substrate, even on removal of the tape.

However, especially in the first experiments, the process' yield was extremely low, and one would have to scan macroscopically large areas to find a micrometer-sized graphene flake (Fig. 3). Needless to say, this search is a practically impossible task for conventional microscopy methods like atomic force microscopy or scanning electron microscopy; realistically only optical microscopy, which relies on the high sensitivity, speed and processing power of the human eye and brain, can do the job. So it came as a pleasant surprise that monolayers of graphite on some substrates $\left(\mathrm{Si} / \mathrm{SiO}_{2}\right.$ with a $300 \mathrm{~nm} \mathrm{\textrm {SiO } _ { 2 }}$ layer, for instance) can produce an optical contrast of up to $15 \%$ for some wavelengths of incoming light. The phenomenon is now well understood (Abergel et al., 2007; Blake et al., 2007) and made $\mathrm{Si} / \mathrm{SiO}_{2}$ with an oxide layer either 100 or $300 \mathrm{~nm}$ thick the substrate of choice

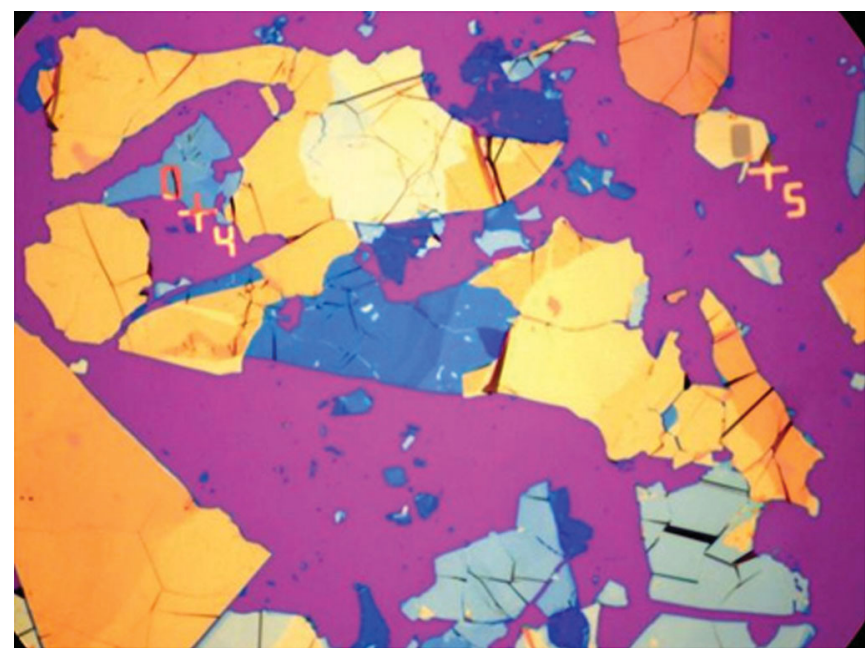

FIG. 3 (color). Thin graphitic flakes on a surface of $\mathrm{Si} / \mathrm{SiO}_{2}$ wafer (300 $\mathrm{nm}$ of $\mathrm{SiO}_{2}$, purple color). The different colors correspond to flakes of differing thicknesses, from $\sim 100 \mathrm{~nm}$ (the pale yellow ones) to a few nanometers (a few graphene layers - the most purple ones). The scale is given by the distance between the lithography marks $(200 \mu \mathrm{m})$. for a number of years for most experimental groups relying on the micromechanical cleavage method of graphene production.

Similar techniques (growth at high temperatures as a part of a 3D system, with subsequent extraction of the 2D part at low temperatures) have been used in other graphene preparation methods. Probably the closest to the micromechanical exfoliation method is chemical exfoliation, which can be traced back to the original work of Professor Brodie (Brodie, 1859) who treated graphite with acids and arrived at "graphon" (or graphite oxide as we now know it). Graphite oxide can be thought of as graphite intercalated with oxygen and hydroxyl groups, which makes it a hydrophilic material and easily dispersible in water. This technique produces extremely thin, sometimes even monolayer, flakes of this material (Ruess and Vogt, 1948; Boehm et al., 1961, 1962a, 1962b; Horiuchi et al., 2004) which can then subsequently be reduced, producing low-quality graphene (Stankovich et al., 2006; Dikin et al., 2007; GomezNavarro et al., 2007; Ruoff, 2008; Park and Ruoff, 2009).

One can imagine an even simpler path for chemical exfoliation. Although graphene is hydrophobic, it can be dispersed in other, mostly organic, solvents (Blake et al., 2008; Hernandez et al., 2008). By repeating the exfoliation and purification (centrifugation) process several times one can obtain $50 \%$ and higher fractions of graphene in suspension.

There are also well-known graphene-growing recipes from surface science. Catalytic cracking of hydrocarbons, or precipitation of dissolved carbon on a metal surface with subsequent graphitization, has long been known to produce high-quality graphene layers (Grant and Haas, 1970; Gall et al., 1985, 1987; Nagashima et al., 1993; Gall et al., 1997; Forbeaux et al., 1998; Affoune et al., 2001; Harigaya and Enoki, 2002). A similar process is the graphitization of excess carbon atoms after sublimation of silicon from the surface of silicon carbide (van Bommel et al., 1975; Berger et al., 2004). One should note that it is also true in these cases that graphene must be a part of the 3D structure, as the underlying substrate aids in quenching the diverging fluctuations at high temperatures.

\section{Other 2D crystals}

Thus far we've been talking mainly about graphene, but the 2D materials family is of course not limited to carbonic crystals, although similar problems are faced when attempts are made to synthesize other $2 \mathrm{D}$ materials. At least two possible methods of obtaining other $2 \mathrm{D}$ crystals come immediately to mind.

One possibility is to apply the same recipes we saw working for graphene to other chemical compounds. Micromechanical or chemical exfoliation can be successfully applied to other layered materials (Fig. 4) such as $\mathrm{Bi}_{2} \mathrm{Sr}_{2} \mathrm{CaCu}_{2} \mathrm{O}_{x}$ (Novoselov et al., 2005a), $\mathrm{NbSe}_{2}$ (Novoselov et al., 2005a), BN (Novoselov et al., 2005a), $\mathrm{MoS}_{2}$ (Novoselov et al., 2005a; Mak et al., 2010), $\mathrm{Bi}_{2} \mathrm{Te}_{3}$ (Teweldebrhan et al., 2010) and other dichalcogenides, and epitaxial growth has been applied to grow monolayers of boron nitride (Nagashima et al., 1995, 1996). As with graphene, the crystal quality of the obtained monolayer samples is very high. Many of the 2D materials conduct 

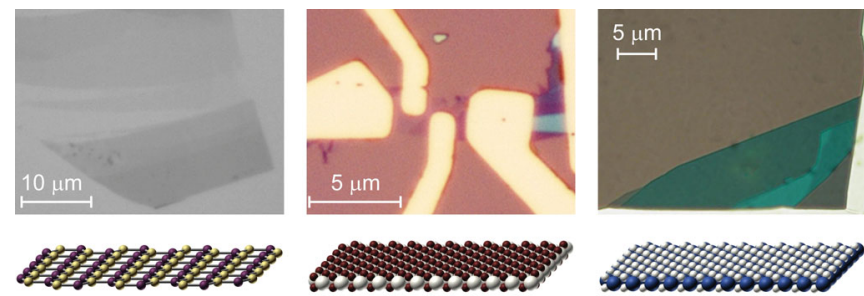

FIG. 4 (color). Optical micrographs of various 2D crystals (top row) and their crystal structures (bottom row). Left: Flakes of boron nitride on top of an oxidized $\mathrm{Si}$ wafer $\left(290 \mathrm{~nm}\right.$ of $\mathrm{SiO}_{2}$, the image taken using narrow band yellow filter, $\lambda=590 \mathrm{~nm}$ ). The central crystal is a monolayer. Center: A device prepared from monolayer and bilayer $\mathrm{NbSe}_{2}$ crystals on an oxidized $\mathrm{Si}$ wafer $(290 \mathrm{~nm}$ of $\mathrm{SiO}_{2}$ ). Right: Flakes of $\mathrm{MoS}_{2}$ on top of an oxidized $\mathrm{Si}$ wafer $\left(90 \mathrm{~nm}\right.$ of $\left.\mathrm{SiO}_{2}\right)$. The piece at the bottom-right corner is a monolayer. Color coding for the crystal structures at the bottom: yellow spheres $=$ boron, purple $=$ nitrogen, large white spheres $=$ niobium, red $=$ selenide, blue $=$ molybdenum, and small white spheres $=$ sulfur.

and even demonstrate field effects (changes of the resistance with gating). The properties of the obtained 2D materials might be very different from those of their 3D precursors. For example, the overlapping between the valence and conduction bands in graphene is exactly zero while it is finite in graphite (Novoselov et al., 2004), and a monolayer of molybdenum disulfide is a direct band semiconductor while the bulk material has an indirect band gap (Mak et al., 2010).

The second approach is to start with an existing 2D crystal and modify it chemically to obtain a new material. One can think of graphene, for instance, as a giant molecule. All the atoms of this molecule are, in principle, accessible for chemical reaction (as opposed to the 3D case where atoms in the interior of the crystal cannot participate in such reactions).

Graphene, due to the versatility of carbon atoms, is a particularly good candidate for such modification. Depending on the environment, the electron configuration of a carbon atom (which has four electrons in the outer shell) might change dramatically, allowing it to bond to two, three, or four other atoms. Bonding between the carbon atoms is exceptionally strong (the strongest materials on Earth are all carbon based), whereas bonding to other species, though stable, can be changed by chemical reactions. To give an example of such versatility: A backbone of two carbon atoms each can accept one, two, or three hydrogen atoms, forming ethyne (also known as acetylene), ethene (also known as ethylene), or ethane, respectively. It is possible to convert any one of those into another by adding or removing hydrogen, thus changing the electron configuration of carbon atoms between so-called $s p, s p^{2}$, and $s p^{3}$ hybridizations.

Carbon atoms in graphene are $s p^{2}$ hybridized, meaning that only three electrons form the strong $\sigma$ bonds and the fourth has a communal use forming the so-called $\pi$ bonds. So, graphene is a zero-overlap semimetal and conducts electricity very well (in contrast to diamond, where each carbon atom is in $s p^{3}$ hybridization and therefore has four neighbors). In that case all four electrons in the outer shell are involved in forming $\sigma$ bonds, so a huge gap appears in the electronic band structure, making diamond an insulator. The

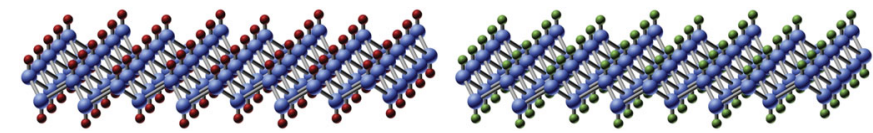

FIG. 5 (color). Chemically modified graphene. One can add different species (such as hydrogen or fluorine) to graphene scaffolding. Carbon atoms are represented by blue spheres.

versatility of carbon atoms, then, gives us an idea of how to create novel 2D crystals: One can attach something to carbon atoms, creating a new material with a different chemical composition and exciting properties.

A wide variety of chemicals can be attached to graphene. So far only two crystallographically ordered chemical modifications of graphene have been predicted and achieved: graphane (when one hydrogen atom is attached to each of the carbon atoms) (Sofo et al., 2007; Elias et al., 2009) and fluorographene (Fig. 5) (Worsley et al., 2007; Cheng et al., 2010; Nair et al., 2010a; Withers et al., 2010). Both derivatives are insulators (exhibiting large band gaps) of very high crystallographic quality and very stable at ambient temperatures (though it should be mentioned that fluorographene generally exhibits more robust properties, probably due to stronger $\mathrm{C}-\mathrm{F}$ bonding in comparison to $\mathrm{C}-\mathrm{H}$ ). Graphane and fluorographene open the floodgates for the chemical modification of graphene and for the appearance of novel two-dimensional atomic crystals with predetermined properties. It would be interesting to see other derivatives, probably filling the gap between graphene and graphane in terms of electrical conductance (the size of the band gap).

Clearly then, the importance of graphene is not only that it has unique properties but also that it has paved the way for, and promoted interest in, the isolation and synthesis of many other 2D materials. We can now talk about a whole new class of materials, 2D atomic crystals, and already have examples with a large variety of properties (from large band-gap insulators to the very best conductors, the extremely mechanically strong to the soft and fragile, and the chemically active to the very inert). Further, many of the properties of these 2D materials are very different from those of their 3D counterparts. Given that, even after 7 years' intensive research, graphene still regularly delivers surprises, it seems reasonable to expect a huge influx of breathtakingly interesting results from the field of 2D atomic crystals.

\section{Out to Spaceland: 2D-based heterostructures}

As mentioned earlier, the properties of $2 \mathrm{D}$ crystals can be very different from their 3D counterparts. Even bilayer graphene (McCann and Fal'ko, 2006; Novoselov et al., 2006) (two graphene layers stacked on top of each other in special, so-called Bernal or A-B, stacking), is remarkably different from graphene. The latter is a zero-overlap semimetal, with linear dispersion relations whenever the bands are parabolic in bilayer graphene, and a gap can be opened in the spectrum if the symmetry between the layers is broken (say by applying electric field between the layers) (McCann, 2006; Ohta et al., 2006; Castro et al., 2007; Oostinga et al., 2007). However, the properties of multilayered materials depend not only on the number of layers (Novoselov et al., 2004; Morozov 

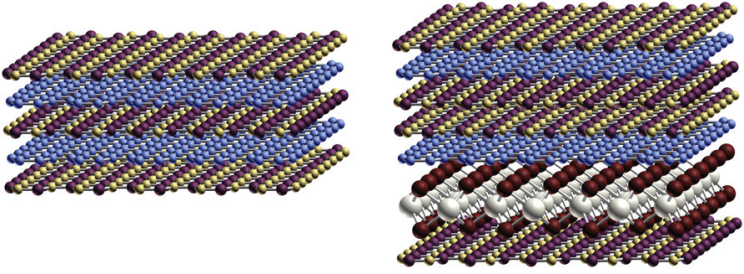

FIG. 6 (color). 2D-based heterostructures. Blue spheres $=$ carbon atoms, $\quad$ yellow $=$ boron, $\quad$ purple $=$ nitrogen, $\quad$ white $=$ niobium, and red $=$ selenide.

et al., 2005) but also on how those layers are stacked. For instance, in the case of graphite, consider Bernal stacking versus rhombohedral versus hexagonal versus turbostratic, and, in bilayer, a small rotation between the individual layers leads to the appearance of van Hove singularities at low energies (Aoki and Amawashi, 2007; dos Santos et al., 2007; Li et al., 2010; Mak et al., 2010).

As we have full control over the $2 \mathrm{D}$ crystals, we can also create stacks of these crystals according to our requirements. Here, we are not merely talking about stacks of the same material: We can combine several different $2 \mathrm{D}$ crystals in one stack. Insulating, conducting, probably superconducting, and magnetic layers can all be combined in one layered material as we wish, the properties of such heterostructures depending on the stacking order and easily tunable.

Thus a completely new world of "materials on demand" is opening up to us. Because the pool of the original 2D crystals is very rich, the properties of such heterostructures can cover a huge parameter space, combining characteristics which previously we would not even dare to think of being found together in one material.

The first members of this huge family are already there. By combining (alternating) monolayers of insulating boron nitride and graphene, one can get weakly coupled graphene layers whose coupling would depend on the number of $\mathrm{BN}$ layers between the graphene planes (Fig. 6). The level of interaction between the graphene planes ranges from tunneling (for single or double BN layers in between) to purely Coulomb (for thicker BN spacers).

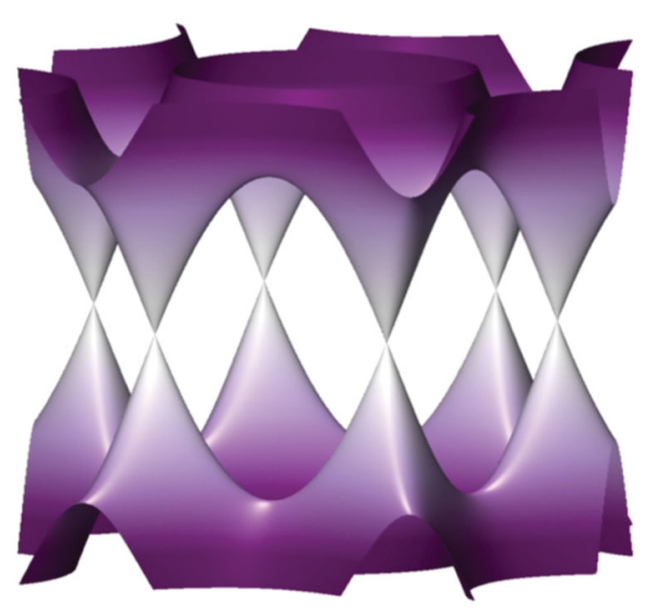

FIG. 7 (color). The low-energy band structure of graphene.

\section{B. Chiral quasiparticles and the electronic properties of graphene}

\section{Linear dispersion relation and chirality}

What really makes graphene special is its electronic properties. Graphene is a zero-overlap semimetal, with valence and conduction bands touching at two points ( $K$ and $K^{\prime}$ ) of the Brillouin zone (Wallace, 1947; McClure, 1957; Slonczewski and Weiss, 1958) (Fig. 7). This is a consequence of the hexagonal symmetry of graphene's lattice (which is not one of the Bravais lattices): It has two atoms per unit cell and can be conceptualized as two interpenetrating triangular lattices. The $p_{z}$ orbitals from the carbon atoms hybridize to form $\pi$ and $\pi^{*}$ bands, whose crossing at the $K$ and $K^{\prime}$ points guarantees a gapless spectrum with linear dispersion relation. Thus, the absence of a gap between the valence and conduction bands in graphene makes for a very robust phenomenon and is a consequence of the symmetry between the sublattices (Slonczewski and Weiss, 1958) (in boron nitride, where the symmetry between the sublattices is broken (one consists of boron, another of nitrogen), a large gap is opened in the electronic spectrum (Novoselov, 2007).

The linear dispersion relation already makes graphene special, but there is more to it than that. States in the valence and conduction bands are essentially described by the same spinor wave function, so electrons and holes are linked via charged conjugation. This link implies that quasiparticles in graphene obey chiral symmetry, similar to that which exists between particles and antiparticles in quantum electrodynamics (QED). This analogy between relativistic particles and quasiparticles in graphene is extremely useful and often leads to interesting interpretations of many phenomena observed in experiment (Neto et al., 2006).

\section{The Klein paradox}

Probably the most striking result of the quasiparticles' chiral symmetry is the prediction (Katsnelson et al., 2006) and observation (Gorbachev et al., 2008; Young and Kim, 2009) of the Klein paradox in graphene [to explore which the $p-n$ junction is a natural venue (Cheianov and Fal'ko, 2006a)]. The paradox refers (Klein, 1929; Sauter, 1932) to the enhanced tunneling probability of a relativistic particle, which approaches unity as the height of the potential barrier exceeds $2 m_{0} c^{2}$ (where $m_{0}$ is the rest mass of the particle and $c$ is the speed of light) and is exactly 1 for massless particles. It can be seen as a result of suppressed backscattering (massless relativistic particles, such as photons, always move with constant velocity - the speed of light-whereas backscattering requires velocity to become zero at the turning point) or as particle-antiparticle pair production and annihilation due to the Schwinger (Schwinger, 1951) mechanism in the areas of high electric field.

The Klein paradox for chiral quasiparticles in graphene leads to perfect electron-to-hole conversion at a potential barrier, and an equal to unity probability of tunneling through such a barrier at least for the normal incidence (Cheianov and Fal'ko, 2006a; Katsnelson et al., 2006; Cheianov and Altshuler, 2007; Beenakker, 2008). This guarantees the absence of localization (Ostrovsky et al., 2006; Bardarson et al., 2007) and finite minimum conductivity (Geim and 


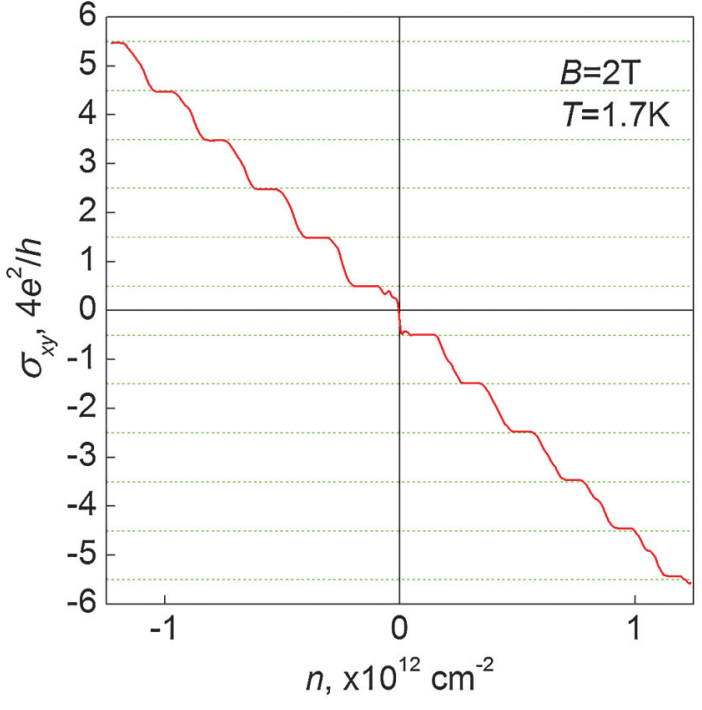

FIG. 8 (color). Hall conductivity as a function of the carrier concentration.

Novoselov, 2007), even in relatively disordered graphene, which - in the limit of nominally zero carrier concentrationsplits into electron-hole puddles (Martin et al., 2008). The absence of backscattering, which leads to the Klein paradox, also ensures that quantum (interference) corrections to the conductivity are positive (at least if we neglect the intervalley scattering and the effect of trigonal warping) (McCann et al., 2006; Castro Neto et al., 2009), resulting in weak antilocalization which has indeed been observed experimentally (Morozov et al., 2006; Tikhonenko et al., 2008).

\section{Half-integer quantum Hall effect}

The charge conjugation symmetry between electrons and holes also guarantees that there should always be an energy level exactly at $E=0$. In the magnetic field this symmetry results in a sequence of the Landau levels as $E_{n}=$ $\pm \sqrt{2 e \hbar v^{2} B\left(n+\frac{1}{2} \pm \frac{1}{2}\right)}$ (here $e$ is an electron charge, $\hbar$ the Plank constant, $B$ the magnetic field, $v$ the Fermi velocity, and $n=0,1,2, \ldots)$, rather different from that for normal massive particles. The $\pm 1 / 2$ term is related to the chirality of the quasiparticles and ensures the existence of two energy levels (one electronlike and one holelike) at exactly zero energy, each with degeneracy 2 times smaller than that of all the other Landau levels (McClure, 1960; Semenoff, 1984; Haldane, 1988; Shon and Ando, 1998; Zheng and Ando, 2002; Gusynin and Sharapov, 2005; Peres et al., 2006).

Experimentally such a ladder of Landau levels exhibits itself in the observation of a "half-integer" quantum Hall effect (Novoselov et al., 2005b; Zhang et al., 2005) (Fig. 8). The 2-times smaller degeneracy of the zero Landau level is revealed by the $\pm \frac{1}{2}\left(4 e^{2} / h\right)$ plateaus in Hall conductivity at filling factors \pm 2 . Furthermore, due to linear dispersion relation and the relatively high value of the Fermi velocity $\left(v \approx 10^{6} \mathrm{~m} / \mathrm{s}\right)$, the separation between the zero and first Landau levels is unusually large (it exceeds the room temperature even in a modest magnetic field of $1 \mathrm{~T}$ ). This, in conjunction with the low broadening of the zero Landau level
(Giesbers et al., 2007), makes it possible to observe the quantum Hall effect even at room temperatures (Novoselov et al., 2007). This is rather exciting news for people working in metrology, as it should allow a much simpler realization of quantum resistance standard (no need for ultralow temperatures), an idea which has recently been supported by several experiments (Giesbers et al., 2008; Tzalenchuk et al., 2010).

\section{Effect of mechanical deformation}

It is important to remember that graphene is not just another 2D electronic system, similar to electrons on the surface of silicon in a metal-oxide-semiconductor field-effect transistor (MOSFET) or in 2D quantum wells in GaAs/ $\mathrm{AlGaAs}$ heterostructures. Graphene is a truly 2D atomic crystal as well as having electronic properties as in 2D. Essentially the thinnest possible fabric, it can be easily deformed mechanically and can be stretched (Bunch et al., 2007, 2008; Lee et al., 2008; Ni et al., 2008; Huang et al., 2009; Kim et al., 2009; Mohiuddin et al., 2009), compressed (Tsoukleri et al., 2009), folded (Hiura et al., 1994; Li et al., 2010), rippled (Bao et al., 2009) and even torn into pieces (Sen et al., 2010). Needless to say, each of these mechanical manipulations results in strong changes to the electronic structure (Kim and Neto, 2008; Pereira and Neto, 2009; Pereira et al., 2009).

Furthermore, it can be demonstrated that strain is equivalent to the local magnetic field (of opposite directions for quasiparticles in the $K$ and $K^{\prime}$ valleys, to preserve the timereversal symmetry) - a phenomenon used to explain the suppression of the weak localization (Morozov et al., 2006; Morpurgo and Guinea, 2006) and additional broadening of all but zeroth Landau levels (Giesbers et al., 2007). In principle, one can imagine engineering strain distribution of a special geometry so that the electronic band structure would be modified as if constant magnetic field was being applied to a particular area of the sample (Guinea et al., 2010, 2010). Since graphene is mechanically strong and very elastic (Lee et al., 2008), the strains applied (and thus the pseudomagnetic fields which would be generated) can be extremely large, resulting in the opening of sizable gaps in the electronic spectrum (Levy et al., 2010). This allows us to talk about a completely new and unexplored direction in electronics: strain engineering of electronic structure (Pereira and Neto, 2009) and valleytronics (Rycerz et al., 2007; Martin et al., 2008; Low and Guinea, 2010).

\section{Graphene optics}

Can one expect anything interesting from the optical properties of graphene? Rather counterintuitively, despite being only one atom thick, graphene absorbs quite a large fraction of light. In the infrared limit the absorption coefficient is exactly $\pi \alpha \approx 2.3 \%$ (where $\alpha=e^{2} / \hbar c$ is the fine structure constant), and the corrections to this number in the visible range of the spectrum are less than 3\% (Kuzmenko et al., 2008; Li et al., 2008; Mak et al., 2008; Nair et al., 2008). Such a significant absorption coefficient makes it possible to see graphene without the use of a microscope; thus, one can observe (literally) the most fundamental constant of this Universe with the naked eye. At higher frequencies the 
absorption becomes even larger, reaching $10 \%$ due to the presence of the van Hove singularities at the zone edge (Yang et al., 2009; Kravets et al., 2010).

By changing the carrier concentration, one can shift the position of the Fermi level and change graphene's optical absorption due to Pauli blocking (Li et al., 2008). Since the density of states in graphene is relatively low (at least in the vicinity of the Dirac point), even electrostatic gating can be enough to shift the Fermi level as high as a few hundred meV (Pachoud et al., 2010), so the Pauli blocking happens in the visible range of the spectrum. By executing such strong gating on several tens of graphene layers in series, it would be possible to control the light transmission in such structures to a large extent, an observation which might be promising for novel photonic devices.

\section{Bilayer graphene}

Although the addition of one layer on top of graphene is all that is needed to arrive at bilayer graphene, the properties of the latter are not simply twice those of the monolayer crystal; this is one of those cases where "one plus one is greater than two." Bilayer graphene is remarkably different-sometimes even richer in its properties than its monolayer cousin-and fully deserves to be called a different material in its own right.

Two graphene layers, when placed together, do not like to lie exactly one on top of each other with each atom having a counterpart in the adjacent layer (unlike boron nitride, which does exactly that). Instead, bilayer graphene is mostly found (Mak et al., 2010) in so-called A-B or Bernal stacking (Bernal, 1924) (named after the famous British scientist John Desmond Bernal, one of the founders of x-ray crystallography, who determined the structure of graphite in 1924). In such an arrangement, only half of the carbon atoms have a neighbor in another layer and the other half do not (and so are projected right into the middle of the hexagon) (Fig. 9). The quantum-mechanical hopping integral between the interacting atoms (generally called $\gamma_{1}$ ) is on the order of $300 \mathrm{meV}$, which gives rise to a pair of high-energy electronic subbands (Dresselhaus and Dresselhaus, 2002; McCann and Fal'ko, 2006; Novoselov et al., 2006). The offset from the zero energy (the position of the Fermi level in undoped bilayer

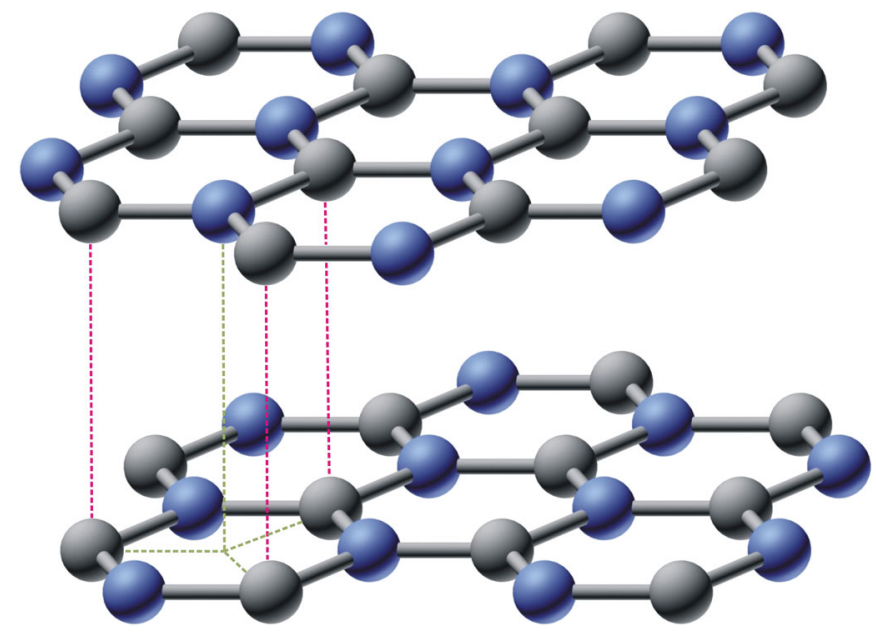

FIG. 9 (color). Crystal structure of bilayer graphene.

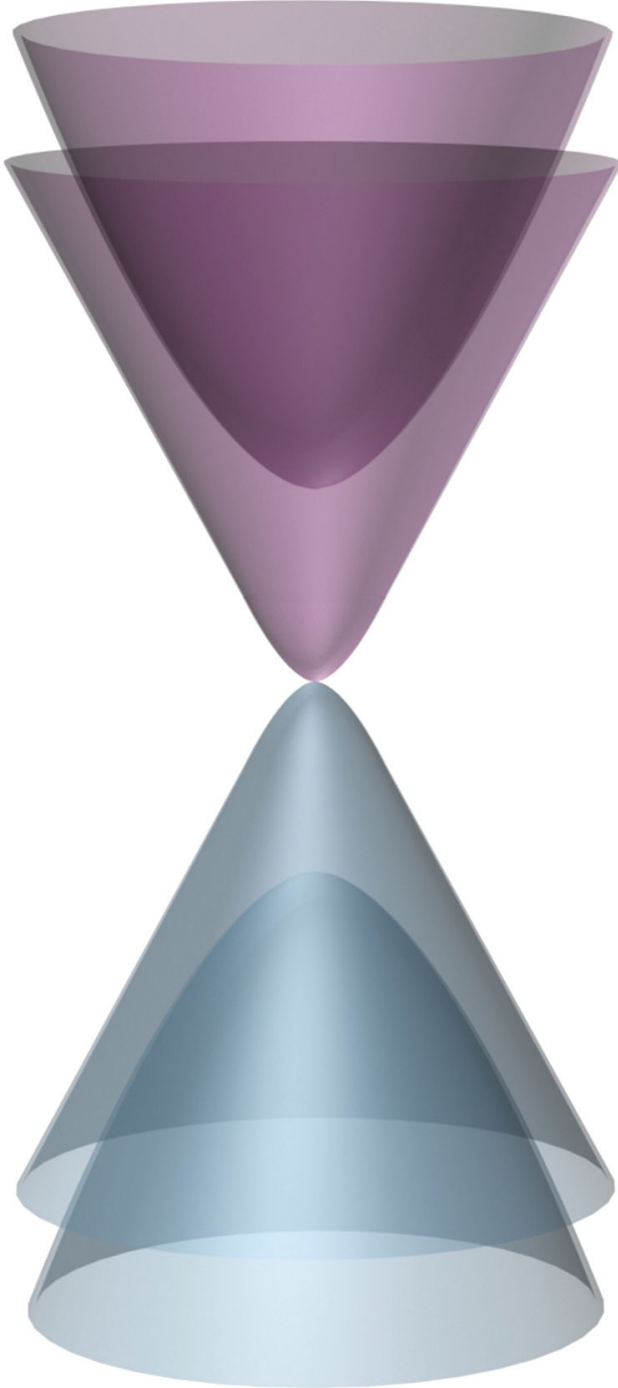

FIG. 10 (color). The band structure of bilayer graphene in the vicinity of the $K$ point.

graphene) is exactly $\gamma_{1}$, so these subbands do not contribute to electronic transport unless a very high level of doping is achieved [though these subbands can easily be observed in optical experiments (Kuzmenko et al., 2009a, 2009b)] (Fig. 10).

The noninteracting atoms give rise to low-energy bands which are still crossing at zero energy (as in graphene), but are parabolic, Fig. 10. The symmetry between the layers is analogous to the sublattice symmetry in monolayer graphene, and it guarantees the chiral symmetry between electrons and holes. Thus we have a new type of quasiparticle in graphene-massive chiral fermions-which does not have an analogy in QED (McCann and Fal'ko, 2006; Novoselov et al., 2006). Similarly to graphene, the chirality reveals itself in the unusual quantum Hall effect. The sequence of the Landau levels in the magnetic field is now $E_{N}=$ $\pm \hbar \omega_{c} \sqrt{N(N-1)}$; here $\omega_{c}=e B / m^{*}$ is the cyclotron frequency and $m^{*}=\gamma_{1} / 2 v^{2}$ is the cyclotron mass. It is easy to see that two Landau levels exist at zero energy $(N=0$ and $N=1$ ), which again ensures a peculiar sequence of the Hall plateaus and metallic behavior in the limit of zero filling 
factor (at least if we neglect the many-body effects) (McCann and Fal'ko, 2006; Novoselov et al., 2006).

As has been said, the chiral symmetry in monolayer and bilayer graphene is protected by the symmetry between the sublattices. In the case of graphene it is rather difficult to break this symmetry-one would have to diligently apply a certain potential to atoms which belong to one sublattice only while applying different potential to another sublattice-but in bilayer graphene it is possible to do just that. By applying a gate voltage or by chemically doping from only one side, we can discriminate between the layers and thus between the sublattices (breaking the inversion symmetry). This results in lifting the chiral symmetry and opening a gap in the spectrum. Both strategies have been implemented in experiment and yielded a rather striking result: A gap as large as $0.5 \mathrm{eV}$ could be opened (Ohta et al., 2006; Castro et al., 2007; Oostinga et al., 2007; Kuzmenko et al., 2009a, 2009b; Zhang et al., 2009; Castro et al., 2010). Thus bilayer graphene is a rare case of a material where the band gap can be directly controlled by (and its size directly proportional to) the electric field applied across the layers.

As the quality of bilayer graphene samples improves (Bolotin et al., 2008; Du et al., 2008; Feldman et al., 2009), we will see more and more interesting properties in them. Topological transitions at low electron energies (Feldman et al., 2009), excitonic effects (Min et al., 2008) and topological one-dimensional states (Martin et al., 2008) are among those which can be expected.

\section{Graphene applications}

Fans of American sitcom The Big Bang Theory (splendidly produced by Chuck Lorre) might recall the episode "The Einstein Approximation" in which string theorist Sheldon tries to resolve "the graphene problem": Why do quasiparticles in graphene behave like massless Dirac fermions?

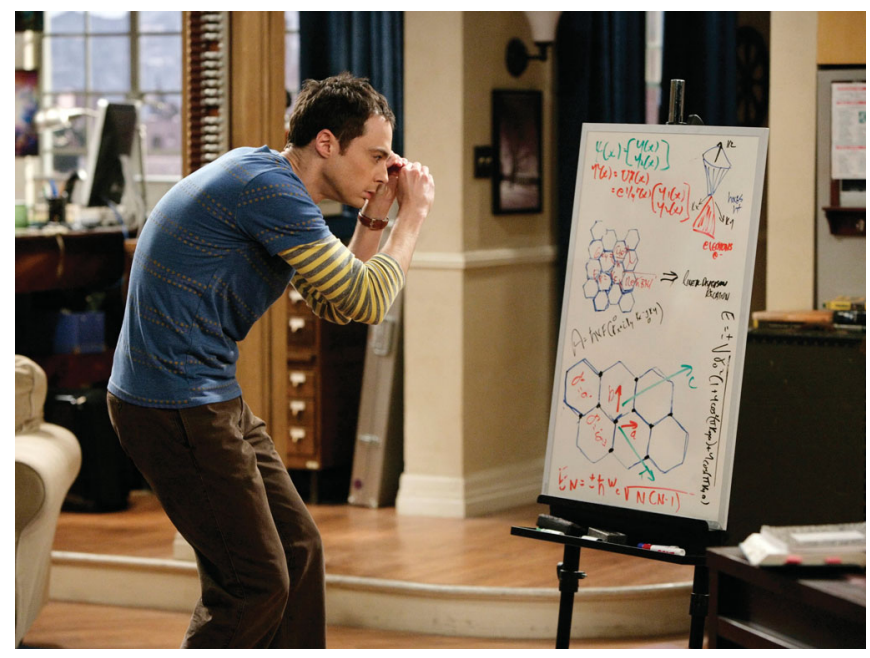

FIG. 11 (color). Dr. Sheldon Cooper (Jim Parsons) “...either isolating the terms of his formula and examining them individually or looking for the alligator that swallowed his hand after Peter Pan cut it off." From The Big Bang Theory, series 3, episode 14 "The Einstein Approximation." Photo: Sonja Flemming/CBS (C2010 CBS Broadcasting Inc.
(Fig. 11) The whole crew is great as usual, particularly actor Jim Parsons' grotesquely brilliant depiction of the tough but enjoyable process of searching for a solution to a scientific problem. It is also probably the best episode from a physics point of view (thanks to Chuck Lorre, other writers, and the scientific advisor David Saltzberg), as-unusually-the whole plot hinges on the scientific problem, rather than this serving merely to link its parts (the only other example I can recall of such an episode is the one about the paper on supersolid). I'd like to think that the reason for this is the simple and appealing physics of graphene, which is Sheldon sophisticated, Penny beautiful, Raj exotic, Leonard practical, and Howard intrusive. On the day the episode was shot Professor Saltzberg wrote in his blog, “... graphene has captured the imagination of physicists with its potential applications," and, in fact, graphene applications are already here.

The point of this paragraph is not to advertise The Big Bang Theory but to demonstrate the kind of applications we are expecting from graphene. The fact that one of the first practical uses of this material was not in a high-expectation, predictable field such as transistors or photonics, but instead in the entertainment industry indicates its great potential and versatility. Indeed, in graphene we have a unique combination of properties which are not seen together anywhere else: conductivity and transparency, mechanical strength and elasticity. Graphene can successfully replace many materials in a great number of existing applications, but I would also like to see things going in the other direction, with the unique combination of properties found in graphene inspiring completely new applications.

\section{Graphene support}

I would like to start by talking about relatively simple graphene devices for quite a minute market: graphene support to study biological and other samples in transmission electron microscopy (TEM) (Wilson et al., 2009; Nair et al., 2010b; Pantelic et al., 2010). It is appealing for the simple reason that graphene membranes are already available on the market and are sold by several companies in both Europe and the U.S.

Graphene is an ultimately thin, ultimately conductive, ultimately mechanically strong, and crystallographically ordered material, and it would be strongly beneficial to use it as a support for nano-objects when observing them in TEM. Its mechanical strength provides rigidity and ease in sample preparation, and it has a very high radiation damage threshold (on the order of $80 \mathrm{keV}$ ). High conductivity eliminates the problem of charging of the support. As it is only one atom thick (and also made of a very light element), graphene ensures the highest possible contrast (one can only go higher in contrast if suspending the object). Finally, because it is highly crystallographically ordered, graphene produces few diffraction spots, and those that do appear can be easily filtered out, leaving the image completely unperturbed by the presence of support. Although graphene is already quite compatible with biomolecules, it could also be functionalized to achieve a certain surface potential (for example, changed from being hydrophobic to hydrophilic). Chemical modification of graphene is already well developed, but there are 
a

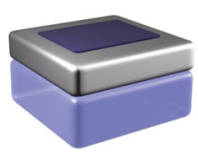

$b$

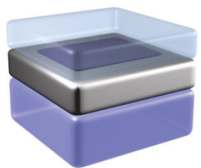

e

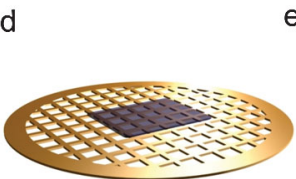

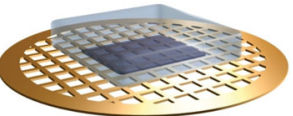

f

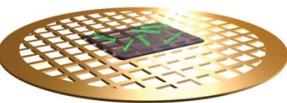

FIG. 12 (color). Production of graphene membranes for TEM support application. Graphene, grown on metal (a) is covered with a layer of plastic (b). The sacrificial metal layer is etched away and graphene on plastic is fished on a standard TEM grid (c). Upon the removal of the plastic layer (d) the graphene membrane can be exposed to a solution of biomolecules (e) which adsorb on the surface of graphene (f) and can be studied in a TEM.

still a large number of opportunities available in this area (Ruoff, 2008; Loh et al., 2010).

Initially, free-standing graphene membranes were produced from exfoliated graphene (Meyer et al., 2007a, 2007b) and required several lithography steps during their manufacture. With the availability of chemical-vapordeposition (CVD) grown graphene ( $\mathrm{Li}$ et al., 2009; Reina et al., 2009), the technique became dramatically simpler, enabling production on an industrial scale. Graphene, epitaxially grown on the surface of a metal (either by precipitation of the dissolved carbon upon cooling or by direct catalytic cracking of the hydrocarbons on the hot surface of the metal, with subsequent graphitization), is covered by a sacrificial layer of plastic. The underlying metal is then removed by etching and the plastic film (with graphene attached) can be transferred onto practically any surface. It can be placed, for example, on a metal grid with holes typically the size of a few microns, where-upon the removal of the sacrificial plastic film - a free-standing graphene membrane is formed (Fig. 12). This entire process is very reproducible and can result in a large total area of graphene membranes.

\section{Transparent conductive coating}

Another area which should benefit significantly from the availability of CVD-grown graphene is transparent conductive coating. Graphene is unusually optically active (Kuzmenko et al., 2008; Li et al., 2008; Nair et al., 2008; Kravets et al., 2010) and absorbs a rather large fraction of incoming light for a monolayer (2.3\%), but this is still significantly smaller than the typical absorption coefficient which could be achieved with a more traditional transparent conductive coating material (Granqvist, 2007). In conjunction with its low electrical resistivity, high chemical stability and mechanical strength, this absorption coefficient makes graphene an attractive material for optoelectronic devices.

Transparent conductors are an essential part of many optical devices, from solar cells to liquid crystal displays and touch screens. Traditionally metal oxides or thin metallic films have been used for these purposes (Granqvist, 2007), but with existing technologies often complicated (thin metallic films require antireflection coating, for example) and expensive (often using noble or rare metals), there has been an ongoing search for new types of conductive thin films. Furthermore, many of the widely used metal oxides exhibit nonuniform absorption across the visible spectrum and are chemically unstable; the commonly used indium tin oxide $\left(\mathrm{In}_{2} \mathrm{O}_{3}: \mathrm{Sn}\right)$, for instance, is known to inject oxygen and indium ions into the active media of a device.

Graphene avoids all of these disadvantages. Moreover, it has recently been demonstrated that large areas of graphene can be grown by the CVD method (Kim et al., 2009; Li et al., 2009; Reina et al., 2009) and transferred onto practically any surface. Prototype devices (solar cells and liquid crystal displays) which use graphene as a transparent conductive coating have already been created (Blake et al., 2008; Wang et al., 2008).

\section{Graphene transistors}

Even the very first graphene field effect transistors demonstrated remarkable quality: prepared using rather humble methods in poorly controlled environments, they still showed reasonably high quasiparticle mobility (up to $20000 \mathrm{~cm}^{2} / \mathrm{V} \mathrm{s}$ ) (Novoselov et al., 2004, 2005a) (Fig. 13). Although the role of different scattering mechanisms is still debated (Ando and Nakanishi, 1998; Ando, 2006; Cheianov and Fal'ko, 2006b; Nomura and MacDonald, 2006; Ostrovsky et al., 2006; Peres et al., 2006; Adam et al., 2007; Nomura and MacDonald, 2007; Stauber et al., 2007; Chen et al., 2008; Katsnelson and Geim, 2008; Morozov et al., 2008; Ponomarenko et al., 2009; Ni et al., 2010), the careful elimination of Coulomb and resonant scatterers, as well as ripples, has allowed the achievement of mobilities of over $10^{6} \mathrm{~cm}^{2} / \mathrm{V} \mathrm{s}$ at low temperatures (Castro et al.) in freestanding devices (Bolotin et al., 2008; Du et al., 2008), and offers hope that values above $10^{5} \mathrm{~cm}^{2} / \mathrm{V} \mathrm{s}$ can be achieved even at ambient temperatures (Dean et al., 2010).

Such characteristics make graphene field effect transistors extremely promising for high-frequency applications (Lin et al., 2009; Moon et al., 2009). Additional benefits are also emerging from very favorable electrostatics of 2D films

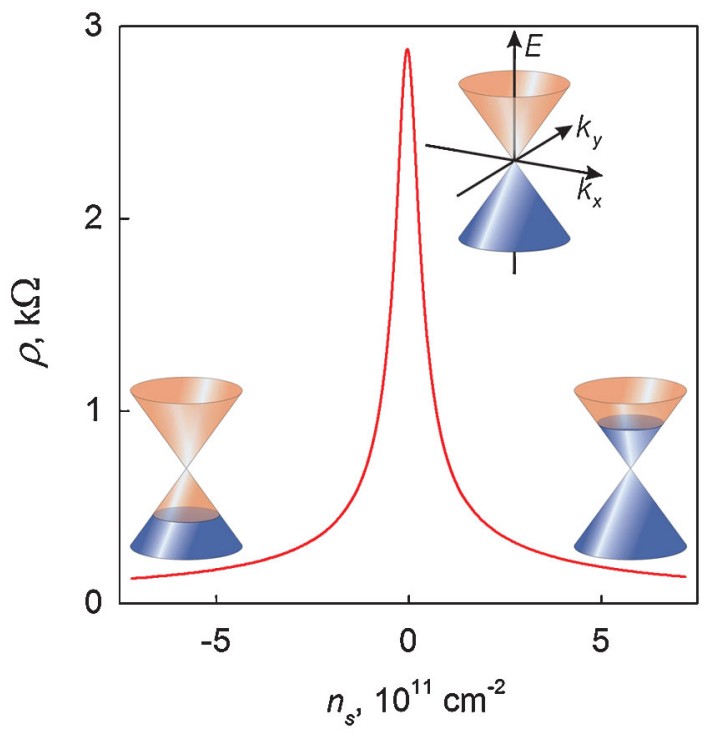

FIG. 13 (color). Ambipolar field effect in graphene. 
and high Fermi velocity (which is important in ballistic regimes). Even when using graphene with rather modest mobility $\left(\sim 10^{3} \mathrm{~cm}^{2} / \mathrm{V} \mathrm{s}\right)$ current gain has been achieved at frequencies as high as $100 \mathrm{GHz}$ for $240 \mathrm{~nm}$ gate length transistors (power gain for similar devices was achieved below $14 \mathrm{GHz}$ ) (Lin et al., 2010), which is better than for Si metal-oxide semiconductor FETs of the same gate length.

The situation is not as bright for integrated circuits. The Klein paradox ensures a finite minimum conductivity for graphene (of the order of $4 e^{2} / h$ ) even within the limit of nominally zero carrier concentration. This is definitely too high for applications in logic elements, as it leads to high "leakage" current in the "off" state and limits the possible on/off ratio of such transistors to about $10^{3}$ even in very favorable circumstances.

There are several possible tricks one can play to increase the on/off ratio of graphene transistors, however. One is to utilize low-dimensional graphene nanostructures such as graphene nanoribbons (Han et al., 2007), quantum dots (Ponomarenko et al., 2008), and single electron transistors (Ponomarenko et al., 2008; Stampfer et al., 2008), where a band gap can be engineered due to quantum confinement or Coulomb blockade. The smallest quantum dots (a few nanometers in size) demonstrate a significant gap on the order of a few hundred $\mathrm{meV}$, which is enough for such transistors to achieve an on/off ratio on the order of $10^{5}$ even at room temperatures (Ponomarenko et al., 2008). The strong carboncarbon bonds ensure the mechanical and chemical stability of such devices, which also can pass a significant current without diminishing their properties. Basically, we can think of it as top-down molecular electronics-one nanometer-sized graphene quantum dot contains only $\sim 10^{2}$ atoms. The major problem with implementing such quantum dots would be the limits of modern lithographic techniques, which do not currently allow true nanometer resolution. Also, one would have to control the roughness and chemistry of the edges with atomic precision, which is also beyond the capabilities of modern technology.

Although modern microelectronics relies on lithographic techniques, one can imagine using other approaches to form nanostructures which would eventually allow reproduction of fine details far beyond the resolution of lithography. One promising method would be the use of the self-organization properties of chemical reactions. Graphene nanostructures could be formed, for instance, by fluorination of the supposed-to-be-insulating parts. Partial fluorination or hydrogenation can result in the formation of self-organized structures on a graphene surface (Chernozatonskii et al., 2007; Shytov et al., 2009), which, in principle, could be used to modify its transport and optical properties.

The other possible way to open a gap in the spectrum of quasiparticles in graphene is to use chemically modified graphene (Worsley et al., 2007; Elias et al., 2009; Cheng et al., 2010; Nair et al., 2010a; Withers et al., 2010), where the $\pi$ electrons are participating in the covalent bond with foreign atoms attached to the carbon scaffolding. One could also use bilayer graphene, as a gap can be opened by applying a potential difference between the two layers (Ohta et al., 2006; Castro et al., 2007; Oostinga et al., 2007; Kuzmenko et al., 2009a, 2009b; Zhang et al., 2009; Castro et al., 2010).
An on/off ratio of 2000 has recently been achieved in dualgated devices at low temperatures (Xia et al., 2010).

\section{Graphene composites}

The unique combination of graphene's electronic, chemical, mechanical, and optical properties can be utilized in full in composite materials. It is also relatively easy to prepare graphene for such an application: One can either use the direct chemical exfoliation of graphene (Blake et al., 2008; Hernandez et al., 2008), which allows a rather high yield of graphene flakes in a number of organic solvents, or go through an oxidation process to prepare graphite oxidewhich can be easily exfoliated in water-with subsequent reduction in a number of reducing media (Stankovich et al., 2006).

The strongest and simultaneously one of the stiffest known materials, with a Young's modulus of $1 \mathrm{TPa}$, graphene is an ideal candidate for use as a reinforcement in highperformance composites (Gong et al., 2010). There is a huge advantage in its being exactly one atom thick: It cannot cleave, giving it the maximum possible strength in the out-ofplane direction. Its high aspect ratio also allows graphene to act as an ideal stopper for crack propagation. As for interaction with the matrix - the central issue for all nanocomposite fillers such as carbon fiber or carbon nanotubes-chemical modification of the surface or edges may significantly strengthen the interface between the graphene and a polymer.

Using chemical derivatives of graphene would not only broaden the range of possible matrices but also widen the functionality of the possible composites. Given that the mechanical strength of fluorographene is only slightly smaller than that of pristine graphene (Nair et al., 2010a), one can obtain composites with similar mechanical properties but a range of other characteristics, from optically transparent to opaque, and from electrically conductive to insulating.

We should also benefit strongly from the possibility of optically monitoring the strain in graphene (Ni et al., 2008; Huang et al., 2009; Mohiuddin et al., 2009; Gong et al., 2010). The Raman spectrum of graphene (Ferrari et al., 2006; Ferrari, 2007; Malard et al., 2009) changes significantly with strain, so even mechanical distortions of a fraction of a percent can be easily detected. As the stress transfer between graphene and polymer is reasonably good (Gong et al., 2010), and graphene's Raman signal is very strong (the strongest peaks in the Raman spectrum of graphene are due to either single or double resonant processes), one can easily detect stress buildup in the composite material under investigation by monitoring the position of the Raman peaks.

\section{Other applications}

It is impossible to review all the potential applications of graphene in one lecture: With practically all the properties of this 2D crystal superior to those in other materials, and the combination of these properties unique, we are limited only by our own imaginations. In terms of electronic properties it is worth mentioning ultimately sensitive gas detectors (Schedin et al., 2007) (graphene, being surface without bulk, can detect a single foreign molecule attached to it) and variable quantum capacitors (Chen and Appenzeller, 
2008; Ponomarenko et al., 2010). In photonics, ultrafast photodetectors (Xia et al., 2009) (utilizing the high mobility and high Fermi velocity of quasiparticles in graphene) and extremely efficient mode lockers (Zhang et al., 2009) must be noted. Additionally, its unprecedented mechanical strength and high crystallographic quality allow one to use graphene to provide the perfect gas barrier (Bunch et al., 2008) and strain gauges (Kim et al., 2009).

\section{ACKNOWLEDGMENTS}

The huge diversity of graphene properties researched and experiments undertaken was only possible thanks to a large and friendly community, which is still growing day by day. I would really like to thank each and every member for their interaction, for teaching me new techniques, and for the excitement I feel every morning opening cond-mat (often tinged with disappointment, admittedly, that I haven't done it first!). Unfortunately it is impossible to name everybody, so I will limit myself to just my closest collaborators and the leaders of the groups. I'm most grateful to Andre Geim, who's been a teacher, colleague, and friend to me over the years. Universities teach us physics, mathematics, chemistry, and tens of other disciplines, but each of us has to work out for himself how to do science. I feel extremely lucky to have worked for all these years alongside such a fantastic and dedicated scientist and researcher. I have also learned a lot from my colleagues in the condensed matter group, the Centre of Mesoscience \& Nanotechnology and Chernogolovka: Irina Grigorieva, Ernie Hill, Sasha Grigorenko, Fred Schedin, Alexander Zhukov, Yuan Zhang, Cinzia Casiraghi, Ursel Bangert, Ian Kinloch, Bob Young, Helen Gleeson, Stan Gillot, Mark Sellers, Oleg Shkliarevskii, Yurij Dubrovskii, Zhenia Vdovin, Yurii Khanin, Sergey Dubonos, and Vsevolod Gantmacher. Special appreciation goes to Sergey Morozov, one of the key figures in graphene research and a fantastic friend. It is impossible to overstate the contribution of our research students and postdocs: their ingenious, creative, and active research often opening new directions of study. I would like to mention Peter Blake, Rahul Nair, Da Jiang, Leonid Ponomarenko, Daniel Elias, Roman Gorbachev, Sasha Mayorov, Tolik Firsov, Soeren Neubeck, Irina Barbolina, Zhenhua Ni, Ibtsam Riaz, Rahul Jalil, Tariq Mohiuddin, Rui Yang, Tim Booth, Liam Britnell, Sveta Anissimova, Frank Freitag, Vasil Kravets, Paul Brimicombe, Margherita Sepioni, and Thanasis Georgiou. The theoretical support provided by a number of condensed matter theorists around the globe was most valuable, and I only hope that the process was reciprocal and that we guided (and misguided) each other equally. The long list of theorists who contributed strongly to our research includes (but is not limited to) Misha Katsnelson, Antonio Castro Neto, Paco Guinea, Nuno Peres, Volodia Falko, Ed McCann, Leonid Levitov, Dima Abanin, Tim Wehling, Allan MacDonald, Sasha Mirlin, and Sankar Das Sarma. Finally, as I have mentioned already, we benefited enormously from collaboration and competition with and information from the other experimental groups. Primarily I must mention Philip Kim, a great physicist and a very good collaborator. Other experimentalists whose results influenced us include (unfortunately only a partial list) Andrea Ferrari, Eva Andrei, Jannik Meyer, Alexey Kuzmenko, Uli Zeitler, Jan Kees Maan, Jos Giesbers, Robin Nicholas, Michal Fuhrer, Tatiana Latychevskaia, Mildred Dresselhaus, Alberto Morpurgo, Lieven Vandersypen, Klaus Ensslin, and Jonathan Coleman.

\section{REFERENCES}

Abergel, D. S. L., A. Russell, and V.I. Fal'ko, 2007, Appl. Phys. Lett. 91, 063125.

Adam, S., et al., 2007, Proc. Natl. Acad. Sci. U.S.A. 104, 18392.

Affoune, A. M., et al., 2001, Chem. Phys. Lett. 348, 17.

Ando, T., 2006, J. Phys. Soc. Jpn. 75, 074716.

Ando, T., and T. Nakanishi, 1998, J. Phys. Soc. Jpn. 67, 1704.

Aoki, M., and H. Amawashi, 2007, Solid State Commun. 142, 123.

Balandin, A. A., et al., 2008, Nano Lett. 8, 902.

Bao, W.Z., et al., 2009, Nature Nanotech. 4, 562.

Bardarson, J. H., et al., 2007, Phys. Rev. Lett. 99, 106801.

Beenakker, C. W. J., 2008, Rev. Mod. Phys. 80, 1337.

Berger, C., et al., 2004, J. Phys. Chem. B 108, 19912.

Bernal, J.D., 1924, Proc. R. Soc. A 106, 749.

Blake, P., et al., 2007, Appl. Phys. Lett. 91, 063124.

Blake, P., et al., 2008, Nano Lett. 8, 1704.

Boehm, H. P., A. Clauss, and U. Hofmann, 1961, J. Chim. Phys. Physicochim. Biol. 58, 141.

Boehm, H. P., et al., 1962a, Z. Naturforsch. 17B, 150.

Boehm, H. P., et al., 1962b, Z. Anorg. Allg. Chem. 316, 119.

Bolotin, K. I., et al., 2008, Solid State Commun. 146, 351.

Brodie, B. C., 1859, Phil. Trans. R. Soc. London 149, 249.

Bunch, J. S., et al., 2007, Science 315, 490.

Bunch, J. S., et al., 2008, Nano Lett. 8, 2458.

Castro, E. V., et al., 2010a, Phys. Rev. Lett. 105, 266601.

Castro, E. V. et al., 2007, Phys. Rev. Lett. 99, 216802.

Castro, E. V. et al., 2010b, J. Phys. Condens. Matter 22, 175503.

Castro Neto, A. H. et al., 2009, Rev. Mod. Phys. 81, 109.

Chaikin, P., and T. C. Lubensky, 1995, Introduction to Condensed Matter Physics (Cambridge University Press, Cambridge).

Cheianov, V. V., V. Fal'ko, and B. L. Altshuler, 2007, Science 315, 1252.

Cheianov, V. V., and V.I. Fal'ko, 2006a, Phys. Rev. B 74, 041403 (R).

Cheianov, V. V., and V.I. Fal'ko, 2006b, Phys. Rev. Lett. 97, 226801.

Chen, J. H., et al., 2008, Nature Phys. 4, 377.

Chen, Z., and J. Appenzeller, 2008, in IEEE International Electron Devices Meeting, Technical Digest (IEEE, New York), p. 509.

Cheng, S. H., et al., 2010, Phys. Rev. B 81, 205435.

Chernozatonskii, L. A., P. B. Sorokin, and J.W. Bruning, 2007, Appl. Phys. Lett. 91, 183103.

Dean, C. R., et al., 2010, Nature Nanotech. 5, 722.

Dikin, D. A., et al., 2007, Nature (London) 448, 457.

dos Santos, J., N. M. R. Peres, and A. H. Castro, 2007, Phys. Rev. Lett. 99, 256802.

Dresselhaus, M. S., and G. Dresselhaus, 2002, Adv. Phys. 51, 1.

Du, X., et al., 2008, Nature Nanotech. 3, 491.

Ebbesen, T. W., and H. Hiura, 1995, Adv. Mater. 7, 582.

Elias, D. C., et al., 2009, Science 323, 610.

Fasolino, A., J. H. Los, and M. I. Katsnelson, 2007, Nature Mater. 6, 858.

Feldman, B. E., J. Martin, and A. Yacoby, 2009, Nature Phys. 5, 889.

Ferrari, A. C., 2007, Solid State Commun. 143, 47.

Ferrari, A. C., et al., 2006, Phys. Rev. Lett. 97, 187401.

Forbeaux, I., et al., 1998, Surf. Rev. Lett. 5, 193. 
Fujibayashi, Y., 1973, J. Phys. Soc. Jpn. 34, 989.

Fujibayashi, Y., and S. Mizushima, 1973, J. Phys. Soc. Jpn. 34, 281. Gall, N. R., E. V. Rutkov, and A. Y. Tontegode, 1997, Int. J. Mod. Phys. B 11, 1865.

Gall, N. R., et al., 1985, Fiz. Tverd. Tela (Leningrad) 27, 2351.

Gall, N. R., et al., 1987, Surf. Sci. 191, 185.

Gan, Y., W. Y. Chu, and L. J. Qiao, 2003, Surf. Sci. 539, 120.

Geim, A. K., 2009, Science 324, 1530.

Geim, A. K., and K. S. Novoselov, 2007, Nature Mater. 6, 183. Giesbers, A. J. M., et al., 2007, Phys. Rev. Lett. 99, 206803.

Giesbers, A. J. M., et al., 2008, Appl. Phys. Lett. 93, 222109.

Girardeau, M., 1960, J. Math. Phys. (Cambridge, Mass.) 1, 516.

Gomez-Navarro C., et al., 2007, Nano Lett. 7, 3499.

Gong, L., et al., 2010, Adv. Mater. 22, 2694.

Gorbachev, R. V., et al., 2008, Nano Lett. 8, 1995.

Granqvist, C. G., 2007, Solar Energy Mater. Sol. Cells 91, 1529.

Grant, J. T., and T. W. Haas, 1970, Surf. Sci. 21, 76.

Guinea, F., M. I. Katsnelson, and A. K. Geim, 2010, Nature Phys. 6, 30 .

Guinea, F., et al., 2010, Phys. Rev. B 81, 035408.

Gusynin, V.P., and S. G. Sharapov, 2005, Phys. Rev. B 71, 125124.

Haldane, F. D. M., 1988, Phys. Rev. Lett. 61, 2015.

Han, M. Y., et al., 2007, Phys. Rev. Lett. 98, 206805.

Harigaya, K., and T. Enoki, 2002, Chem. Phys. Lett. 351, 128.

Hernandez, Y., et al., 2008, Nature Nanotech. 3, 563.

Hiura, H., et al., 1994, Nature (London) 367, 148.

Horiuchi, S., et al., 2004, Appl. Phys. Lett. 84, 2403.

Huang, M. Y., et al., 2009, Proc. Natl. Acad. Sci. U.S.A. 106, 7304.

Katsnelson, M. I., and A. K. Geim, 2008, Phil. Trans. R. Soc. A 366, 195.

Katsnelson, M. I., K. S. Novoselov, and A. K. Geim, 2006, Nature Phys. 2, 620.

Kim, E. A., and A.H.C. Neto, 2008, Europhys. Lett. 84, 57007.

Kim, K. S., et al., 2009, Nature (London) 457, 706.

Klein, O., 1929, Z. Phys. A 53, 157.

Kravets, V. G., et al., 2010, Phys. Rev. B 81, 155413.

Kuzmenko, A. B., et al., 2008, Phys. Rev. Lett. 100, 117401.

Kuzmenko, A. B., et al., 2009a, Phys. Rev. B 80, 165406.

Kuzmenko, A. B., et al., 2009b, Phys. Rev. B 79, 115441.

Landau, L. D., 1937, Phys. Z. Sowjetunion 11, 26.

Landau, L. D., and E. M. Lifshitz, 1980, Statistical Physics, Part I (Pergamon, Oxford, UK), Vol. 1.

Lee, C., et al., 2008, Science 321, 385.

Levy, N., et al., 2010, Science 329, 544.

Li, G. H., et al., 2010, Nature Phys. 6, 109.

Li, X. S., et al., 2009, Science 324, 1312.

Li, Z. Q., et al., 2008, Nature Phys. 4, 532.

Lieb, E. H., and W. Liniger, 1963, Phys. Rev. 130, 1605.

Lin, Y. M., et al., 2009, Nano Lett. 9, 422.

Lin, Y. M., et al., 2010, Science 327, 662.

Loh, K. P., et al., 2010, J. Mater. Chem. 20, 2277.

Low, T., and F. Guinea, 2010, Nano Lett. 10, 3551.

Lu, X. K., et al., 1999, Appl. Phys. Lett. 75, 193.

Mak, K. F., J. Shan, and T.F. Heinz, 2010, Phys. Rev. Lett. 104, 176404

Mak, K. F., et al., 2008, Phys. Rev. Lett. 101, 196405.

Mak, K. F., et al., 2010, Phys. Rev. Lett. 105, 136805.

Malard, L. M., et al., 2009, Phys. Rep. 473, 51.

Martin, I., Y. M. Blanter, and A. F. Morpurgo, 2008, Phys. Rev. Lett. 100, 196804.

Martin, J., et al., 2008, Nature Phys. 4, 144.

McCann, E., 2006, Phys. Rev. B 74, 161403.

McCann, E., and V. I. Fal'ko, 2006, Phys. Rev. Lett. 96, 086805.

McCann, E., et al., 2006, Phys. Rev. Lett. 97, 146805.
McClure, J. W., 1957, Phys. Rev. 108, 612.

McClure, J. W., 1960, Phys. Rev. 119, 606.

Meric, I., et al., 2008, Nature Nanotech. 3, 654.

Mermin, N. D., 1968, Phys. Rev. 176, 250.

Mermin, N. D., and H. Wagner, 1966, Phys. Rev. Lett. 17, 1133.

Meyer, J. C., et al., 2007a, Nature (London) 446, 60.

Meyer, J. C., et al., 2007b, Solid State Commun. 143, 101.

Min, H. K., et al., 2008, Phys. Rev. B 78, 121401.

Mizushima, S., Y. Fujibayashi, and K. Shiiki, 1971, J. Phys. Soc. Jpn. 30, 299.

Mohiuddin, T. M. G., et al., 2009, Phys. Rev. B 79, 205433.

Moon, J. S., et al., 2009, IEEE Electron Device Lett. 30, 650.

Morozov, S. V., et al., 2005, Phys. Rev. B 72, 201401.

Morozov, S. V., et al., 2006, Phys. Rev. Lett. 97, 016801.

Morozov, S. V., et al., 2008, Phys. Rev. Lett. 100, 016602.

Morpurgo, A.F., and F. Guinea, 2006, Phys. Rev. Lett. 97, 196804.

Nagashima, A., et al., 1993, Surf. Sci. 291, 93.

Nagashima, A., et al., 1995, Phys. Rev. Lett. 75, 3918.

Nagashima, A., et al., 1996, Surf. Sci. 357-358, 307.

Nair, R. R., et al., 2008, Science 320, 1308.

Nair, R. R., et al., 2010a, Small 6, 2877.

Nair, R. R., et al., 2010b, Appl. Phys. Lett. 97, 153102.

Nelson, D., D. R. Piran, and S. Weinberg, 2004, Statistical Mechanics of Membranes and Surfaces (World Scientific, Singapore).

Neto, A. C., F. Guinea, and N. M. R. Peres, 2006, Phys. World 19, 33.

Ni, Z.H., et al., 2008, ACS Nano 2, 2301.

Ni, Z. H., et al., 2010, Nano Lett. 10, 3868.

Nomura, K., and A.H. MacDonald, 2006, Phys. Rev. Lett. 96, 256602.

Nomura, K., and A.H. MacDonald, 2007, Phys. Rev. Lett. 98, 076602 .

Novoselov, K., 2007, Nature Mater. 6, 720.

Novoselov, K. S., et al., 2004, Science 306, 666.

Novoselov, K. S., et al., 2005a, Proc. Natl. Acad. Sci. U.S.A. 102, 10451.

Novoselov, K. S. et al., 2005b, Nature (London) 438, 197.

Novoselov, K. S., et al., 2006, Nature Phys. 2, 177.

Novoselov, K. S., et al., 2007, Science 315, 1379.

Ohashi, Y., et al., 1997, Tanso 180, 235.

Ohta, T., et al., 2006, Science 313, 951.

Oostinga, J. B., et al., 2007, Nature Mater. 7, 151.

Ostrovsky, P. M., I. V. Gornyi, and A. D. Mirlin, 2006, Phys. Rev. B 74, 235443.

Pachoud, A., et al., 2010, Europhys. Lett. 92, 27001.

Pantelic, R. S., et al., 2010, J. Struct. Biol. 170, 152.

Park, S., and R. S. Ruoff, 2009, Nature Nanotech. 4, 217.

Peierls, R.E., 1934, Helv. Phys. Acta 7, 81.

Peierls, R. E., 1935, Ann. Inst. Henri Poincaré 5, 177.

Pereira, V.M., and A.H.C. Neto, 2009, Phys. Rev. Lett. 103, 046801.

Pereira, V. M., A. H. C. Neto, and N. M. R. Peres, 2009, Phys. Rev. B 80, 045401.

Peres, N. M. R., F. Guinea, and A.H. C. Neto, 2006, Phys. Rev. B 73, 125411.

Pólya G., 1921, Math. Ann. 84, 149.

Ponomarenko, L. A., et al., 2008, Science 320, 356.

Ponomarenko, L. A., et al., 2009, Phys. Rev. Lett. 102, 206603.

Ponomarenko, L. A., et al., 2010, Phys. Rev. Lett. 105, 136801.

Reina, A., et al., 2009, Nano Lett. 9, 30.

Ruess, G., and F. Vogt, 1948, Monatsh. Chem. 78, 222.

Ruoff, R., 2008, Nature Nanotech. 3, 10. 
Rycerz, A., J. Tworzydlo, and C. W. J. Beenakker, 2007, Nature Phys. 3, 172.

Sauter, F., 1932, Z. Phys. A 73, 547.

Schedin, F., et al., 2007, Nature Mater. 6, 652.

Schwierz, F., 2010, Nature Nanotech. 5, 487.

Schwinger, J., 1951, Phys. Rev. 82, 664.

Semenoff, G. W., 1984, Phys. Rev. Lett. 53, 2449.

Sen, D., et al., 2010, Small 6, 1108.

Shon, N. H., and T. Ando, 1998, J. Phys. Soc. Jpn. 67, 2421.

Shytov, A. V., D. A. Abanin, and L. S. Levitov, 2009, Phys. Rev. Lett. 103, 016806.

Simpson, C. D., et al., 2002, Chem. Eur. J. 8, 1424.

Slonczewski, J. C., and P. R. Weiss, 1958, Phys. Rev. 109, 272.

Sofo, J. O., A. S. Chaudhari, and G. D. Barber, 2007, Phys. Rev. B 75, 153401.

Stampfer, C., et al., 2008, Appl. Phys. Lett. 92, 012102.

Stankovich, S., et al., 2006, Nature (London) 442, 282.

Stauber, T., N. M. R. Peres, and F. Guinea, 2007, Phys. Rev. B 76, 205423.

Teweldebrhan, D., V. Goyal, and A. A. Balandin, 2010, Nano Lett. 10, 1209.
Tikhonenko, F. V., et al., 2008, Phys. Rev. Lett. 100, 056802.

Tsoukleri, G., et al., 2009, Small 5, 2397.

Tzalenchuk, A., et al., 2010, Nature Nanotech. 5, 186.

van Bommel, A. J., J. E. Crombeen, and A. van Tooren, 1975, Surf. Sci. 48, 463.

Wallace, P. R., 1947, Phys. Rev. 71, 622.

Wang, X., L.J. Zhi, and K. Mullen, 2008, Nano Lett. 8, 323.

Wilson, N. R., et al., 2009, ACS Nano 3, 2547.

Withers, F., M. Dubois, and A. K. Savchenko, 2010, Phys. Rev. B 82, 073403.

Worsley, K. A., et al., 2007, Chem. Phys. Lett. 445, 51.

Xia, F. N., et al., 2009, Nature Nanotech. 4, 839.

Xia, F. N., et al., 2010, Nano Lett. 10, 715.

Yang, L., et al., 2009, Phys. Rev. Lett. 103, 186802.

Young, A. F., and P. Kim, 2009, Nature Phys. 5, 222.

Zhang, H., et al., 2009, Opt. Express 17, 17630.

Zhang, Y. B., et al., 2005, Nature (London) 438, 201.

Zhang, Y. B., et al., 2009, Nature (London) 459, 820.

Zheng, Y., and T. Ando, 2002, Phys. Rev. B 65, 245420. 